\title{
Existence and boundary behavior of weak solutions for Schrödingerean TOPSIS equations
}

Yong Wang ${ }^{1}$, Jianguo Sun ${ }^{1}$, Liang Kou' ${ }^{1}$, Yong Lu²*, Guodong Zhao' ${ }^{1}$ Wenshan Wang ${ }^{1}$ and Qilong Han ${ }^{1}$

"Correspondence: ylu77@qq.com ${ }^{2}$ College of Power and Energy Engineering, Harbin Engineering University, Harbin, 150001, China Full list of author information is available at the end of the article

\begin{abstract}
In this paper, we prove that there exists a weak solution for Schrödingerean technique for order performance by similarity (TOPSIS) equations on cylinders. Meanwhile, the boundary behaviors of it are also obtained via the abstract theory of fuzzy multi-criterion decision making. As the main tools, we use Karamata regular variation theory and the method of upper and lower solutions.
\end{abstract}

Keywords: Schrödingerean TOPSIS equations; boundary behavior; cylinder

\section{Introduction}

Motivated by uncertainty problems, risk measures and the superhedging in finance, Xue established the fundamental theory of Schrödingerean expectation theory (see [1]), where the minimally thin sets associated with a Schrödinger operator are introduced. In the Schrödingerean expectation framework, the notion of the corresponding Schrödingerean stochastic calculus of Itô type were also established (see [2]). As in [3], the set

$$
\Omega \times \mathbf{R}=\left\{P=(X, y) \in \mathbf{R}^{n} ; X \in \Omega, y \in \mathbf{R}\right\}
$$

in $\mathbf{R}^{n}$ is simply denoted by $\mathcal{C}_{n}(\Gamma)$. We call it a cylinder (see [3]). On that basis, the theory and applications of the Schrödingerean TOPSIS equation have been developed rapidly (see $[2,4-11]$ and the references therein).

In this paper, we consider the following Schrödingerean TOPSIS equation:

$$
T(-\triangle)^{s} u+a(P) u(P)=0
$$

in $\mathcal{C}_{n}(\Gamma)$, where $0<s \leq 1$ and the potential $a$ satisfies the following condition:

$$
0<\inf _{P=(r, \Theta) \in C_{n}(\Omega)} a(P)<\lim _{r \rightarrow \infty} a(P)=a_{\infty}<\infty .
$$

Under the Lipschitz assumptions on the potential $a$, Yang (see [11]) has proved the wellposedness of such equations with the fixed-point iteration. Moreover, Liu (see [8]) has studied the Markov chains when coefficients are integral-Lipschitz, Zhang and Wu (see

(c) The Author(s) 2018. This article is distributed under the terms of the Creative Commons Attribution 4.0 International License (http://creativecommons.org/licenses/by/4.0/), which permits unrestricted use, distribution, and reproduction in any medium, provided you give appropriate credit to the original author(s) and the source, provide a link to the Creative Commons license, and indicate if changes were made. 
[9]) considered the modified Laplace equations with some good boundaries, Wang et al. (see [10]) studied stochastic functional differential equations with infinite delay. We can also refer the reader to Miyamoto (see [3]), Chen (see [5] and the references therein).

Let $\alpha>0$ and $1 \leq p<\infty$. Then the weighted weak space $\aleph_{\alpha}^{p}(\Gamma)$ on cylinders can be defined by

$$
\|u\|_{\aleph_{\alpha}^{p}(\Gamma)}:=\left(\int_{\Gamma}|u(y)|^{p} d \wp_{\alpha}(y)\right)^{\frac{1}{q}}<\infty
$$

where $u$ are weak solutions of (1.1) on cylinders, $d \wp_{\alpha}(y)=\operatorname{dist}(y, \partial \Gamma)^{\alpha} d y$ and $1 / p+1 / q=1$. Let $d y$ denote the Lebesgue measure on $\mathbf{R}^{n}$ and $\operatorname{dist}(y, \partial \Gamma)$ denote the Euclidean distance from $z$ to the boundary of $\Gamma$. We let $\aleph_{\alpha}^{p}=\aleph_{\alpha}^{p}\left(\mathcal{C}_{n}(\Gamma)\right)$. Then we can check that $d V_{\alpha}(y)=y_{n}^{\alpha} d y$ in $\mathcal{C}_{n}(\Gamma)$.

Weak spaces are not studied as extensively as their holomorphic counterparts and many results on spaces has been done for bounded domains (see [12, 13]), for example, are good references for holomorphic Bergman spaces. $\aleph_{0}^{p}(\Gamma)$ is studied in [5] and [3, 6] on the setting of upper half-space and bounded smooth domain in $\mathbf{R}^{n}$, respectively. $\aleph_{\alpha}^{p}(B)$, where $B$ is the open unit ball and the upper half plane in $\mathbf{R}^{n}$, are studied in [7] and [1], respectively.

For nonnegative functions $g_{1}$ and $g_{2}$, we often write $g_{1} \leq g_{2}$ or $g_{2} \geq g_{1}$ if $g_{1} \leq c g_{2}$, where $c$ is an inessential positive constant. Also, we write $g_{1} \approx g_{2}$ if $g_{1} \leq g_{2}$ and $g_{2} \leq g_{1}$. Throughout this paper, we shall use the same letter $C$ to denote various constants which may be different from line to line.

\section{Preliminary results}

In this section, we first recall one definition and some previous results about the generalized Poisson kernel and Green function in the half space, which will be available later.

Let $z \in \mathbf{R}^{n}$ and $r>0$. Let $B(y, r)$ denote the open ball in $\mathbf{R}^{n}$. Let $V(B(0,1))$ be the volume of the unit ball in $\mathbf{R}^{n}, w \in \overline{\mathcal{C}_{n}(\Gamma)}, \bar{w}=\left(w^{\prime},-w_{n}\right)$ and $z \in \mathcal{C}_{n}(\Gamma)$. Then the extended Poisson kernel $P(y, w)$ in $\mathcal{C}_{n}(\Gamma)$ can be defined by

$$
P_{z}(w):=P(y, w)=\frac{1}{n V(B(0,1))} \frac{z_{n}+w_{n}}{|z-\bar{w}|^{n}} .
$$

It is easy to see that (see [14] for details and related facts)

$$
\int_{\partial \mathcal{C}_{n}(\Gamma)} P(y, w) d w^{\prime}=1
$$

for each $z \in \mathcal{C}_{n}(\Gamma)$ and for every $w \in \overline{\mathcal{C}_{n}(\Gamma)}$.

Let $\vec{\beta}=\left(\beta_{1}, \beta_{2}, \ldots, \beta_{n}\right)$ be a multi-index with $\beta_{j} \in \mathbf{N} \cup\{0\}$ for $j=1,2, \ldots, n$ and $f$ be a homogeneous polynomial of degree $|\vec{\beta}|+2$. Then we see from (2.1) that

$$
D_{y}^{\vec{\beta}} P(y, w):=D_{z_{1}}^{\beta_{1}} \cdots D_{z_{1}}^{\beta_{1}} P(y, w)=\frac{f(y-\bar{w})}{|z-\bar{w}|^{n+2|| \vec{\beta} \mid+1}},
$$

where $\vec{\beta}=\beta_{1}+\beta_{2}+\cdots+\beta_{n}$.

The following lemma collects so-called Poisson-Schrödinger type estimates (see [4]), which play important roles in our discussions. 
Lemma 2.1 If $\vec{\beta}$ is a multi-index, $u$ is the weak solution of (1.1) and bounded by $M$ on $B(y, r)$, then there exists a positive constant $C$ depending on $\vec{\beta}$ such that

$$
\left|D^{\vec{\beta}} u(y)\right| \leq \frac{C M}{r|\vec{\beta}|+1} .
$$

\section{Main results}

For the rest of this paper, we assume $\alpha>0, p, q \in(0, \infty)$ and $u$ is the weak solution of (1.1).

First we prove that equation (1.1) has at least a weak solution.

Theorem 3.1 If a changes its sign, then (1.1) has at least a weak solution $u_{\lambda}$.

Proof For convenience, let

$$
d_{n}=\left(I-\frac{\mu_{n}}{\sigma_{n}} \mathcal{G}^{*} \mathcal{G}\right) v_{n}
$$

Using Lemma 2.1 it follows that $\left(I-\frac{\mu_{n}}{\sigma_{n}} \mathcal{G}^{*} \mathcal{G}\right)$ is nonexpansive and averaged. Hence,

$$
\begin{aligned}
\left\|t_{n+1}-t_{n}\right\| \leq & \frac{\sigma_{n+1}}{1+\sigma_{n+1}}\left\|d_{n+1}-d_{n}\right\|+\left|\frac{\sigma_{n+1}}{1+\sigma_{n+1}}-\frac{\sigma_{n}}{1+\sigma_{n}}\right|\left\|d_{n}\right\| \\
& +\frac{T}{1+\sigma_{n+1}}\left\{\left(1-\sigma_{n+1}\right) w_{n+1}+\sigma_{n+1} d_{n+1}-\left[\left(1-\sigma_{n}\right) w_{n}+\sigma_{n} d_{n}\right]\right\} \\
& +\left|\frac{1}{1+\sigma_{n+1}}-\frac{1}{1+\sigma_{n}}\right|\left\|T\left[\left(1-\sigma_{n}\right) w_{n}+\sigma_{n} d_{n}\right]\right\| \\
\leq & \frac{\sigma_{n+1}}{1+\sigma_{n+1}}\left\|d_{n+1}-d_{n}\right\|+\left|\frac{\sigma_{n+1}}{1+\sigma_{n+1}}-\frac{\sigma_{n}}{1+\sigma_{n}}\right|\left\|d_{n}\right\| \\
& +\frac{1-\sigma_{n+1}}{1+\sigma_{n+1}}\left\|w_{n+1}-w_{n}\right\|+\frac{\sigma_{n+1}}{1+\sigma_{n+1}}\left\|d_{n+1}-d_{n}\right\|+\frac{\sigma_{n}-\sigma_{n+1}}{1+\sigma_{n+1}}\left\|w_{n}\right\| \\
& +\frac{\sigma_{n+1}-\sigma_{n}}{1+\sigma_{n+1}}\left\|d_{n}\right\|+\left|\frac{1}{1+\sigma_{n+1}}-\frac{1}{1+\sigma_{n}}\right|\left\|T\left[\left(1-\sigma_{n}\right) w_{n}+\sigma_{n} d_{n}\right]\right\| .
\end{aligned}
$$

Moreover,

$$
\begin{aligned}
\left\|d_{n+1}-d_{n}\right\|= & \left\|\left(I-\frac{\mu_{n+1}}{\sigma_{n+1}} \mathcal{G}^{*} \mathcal{G}\right) v_{n+1}-\left(I-\frac{\mu_{n}}{\sigma_{n}} \mathcal{G}^{*} \mathcal{G}\right) v_{n}\right\| \\
\leq & \left\|v_{n+1}-v_{n}\right\| \\
= & \| P_{S_{i}}\left[\left(1-\alpha_{n+1}\right) w_{n+1}-\gamma_{n} \mathcal{G}^{*} \mathcal{G} w_{n+1}\right] \\
& -P_{S_{i}}\left[\left(1-\alpha_{n}\right) w_{n}-\gamma_{n} \mathcal{G}^{*} \mathcal{G} w_{n}\right] \| \\
\leq & \left\|\left(I-\gamma_{n+1} \mathcal{G}^{*} \mathcal{G}\right) w_{n+1}-\left(I-\gamma_{n+1} \mathcal{G}^{*} \mathcal{G}\right) w_{n}+\left(\gamma_{n}-\gamma_{n+1}\right) \mathcal{G}^{*} \mathcal{G} w_{n}\right\| \\
& +\alpha_{n+1}\left\|-w_{n+1}\right\|+\alpha_{n}\left\|w_{n}\right\| \\
\leq & \left\|w_{n+1}-w_{n}\right\|+\left|\gamma_{n}-\gamma_{n+1}\right|\left\|\mathcal{G}^{*} \mathcal{G} w_{n}\right\| \\
& +\alpha_{n+1}\left\|-w_{n+1}\right\|+\alpha_{n}\left\|w_{n}\right\| .
\end{aligned}
$$


Substituting (3.2) in (3.1), we infer that

$$
\begin{aligned}
\left\|t_{n+1}-t_{n}\right\| \leq & \left|\frac{\sigma_{n+1}}{1+\sigma_{n+1}}-\frac{\sigma_{n}}{1+\sigma_{n}}\right|\left\|d_{n}\right\|+\frac{\sigma_{n}-\sigma_{n+1}}{1+\sigma_{n+1}}\left\|w_{n}\right\|+\frac{\sigma_{n+1}-\sigma_{n}}{1+\sigma_{n+1}}\left\|d_{n}\right\| \\
& +\left\|w_{n+1}-w_{n}\right\|+\left|\frac{1}{1+\sigma_{n+1}}-\frac{1}{1+\sigma_{n}}\right|\left\|T\left[\left(1-\sigma_{n}\right) w_{n}+\sigma_{n} d_{n}\right]\right\| \\
& +\left|\gamma_{n}-\gamma_{n+1}\right|\left\|w_{n}\right\|+\alpha_{n+1}\left\|-w_{n+1}\right\|+\alpha_{n}\left\|w_{n}\right\| .
\end{aligned}
$$

By virtue of $\lim _{n \rightarrow \infty}\left(\sigma_{n+1}-\sigma_{n}\right)=0$, it follows that

$$
\lim _{n \rightarrow \infty}\left(\left|\frac{\sigma_{n+1}}{1+\sigma_{n+1}}-\frac{\sigma_{n}}{1+\sigma_{n}}\right|\right)=0 .
$$

Moreover, $\left\{w_{n}\right\}$, and $\left\{v_{n}\right\}$ are bounded, and so is $\left\{d_{n}\right\}$. Therefore, (3.2) reduces to

$$
\lim _{n \rightarrow \infty} \sup \left(\left\|t_{n+1}-t_{n}\right\|-\left\|w_{n+1}-w_{n}\right\|\right) \leq 0
$$

Applying (3.3) and Karamata regular variation theory, we get

$$
\lim _{n \rightarrow \infty}\left\|t_{n}-w_{n}\right\|=0
$$

Combining (3.4) with (3.2), we obtain

$$
\lim _{n \rightarrow \infty}\left\|x_{n+1}-x_{n}\right\|=0
$$

Using the convexity of the norm and (3.5), we deduce that

$$
\begin{aligned}
\left\|w_{n+1}-\hat{w}\right\|^{2} \leq & \left(1-\sigma_{n}\right)\left\|w_{n}-\hat{w}\right\|^{2}+\sigma_{n}\left\|v_{n}-\hat{w}\right\|^{2} \\
\leq & \sigma_{n}\left\|-\alpha_{n} \hat{w}+\left(1-\alpha_{n}\right)\left[w_{n}-\frac{\gamma_{n}}{1-\alpha_{n}} \mathcal{G}^{*} \mathcal{G} w_{n}-\left(\hat{w}-\frac{\gamma_{n}}{1-\alpha_{n}} \mathcal{G}^{*} \mathcal{G} \hat{w}\right)\right]\right\|^{2} \\
\leq & \left(1-\sigma_{n}\right)\left\|w_{n}-\hat{w}\right\|^{2}+\sigma_{n} \alpha_{n}\|-\hat{w}\|^{2}+\left(1-\alpha_{n}\right) \sigma_{n}\left[\left\|w_{n}-\hat{w}\right\|^{2}\right. \\
& \left.+\frac{\gamma_{n}}{1-\alpha_{n}}\left(\frac{\gamma_{n}}{1-\alpha_{n}}-\frac{2}{\rho(\mathcal{G} * \mathcal{G})}\right)\left\|\mathcal{G}^{*} \mathcal{G} w_{n}-\mathcal{G}^{*} \mathcal{G} \hat{w}\right\|^{2}\right] \\
\leq & \left\|w_{n}-\hat{w}\right\|^{2}+\sigma_{n} \alpha_{n}\|-\hat{w}\|^{2} \\
& +\sigma_{n} \gamma_{n}\left(\frac{\gamma_{n}}{1-\alpha_{n}}-\frac{2}{\rho(\mathcal{G} * \mathcal{G})}\right)\left\|\mathcal{G}^{*} \mathcal{G} w_{n}-\mathcal{G}^{*} \mathcal{G} \hat{w}\right\|^{2}
\end{aligned}
$$

which implies that

$$
\begin{aligned}
\left\|\mathcal{G}^{*} \mathcal{G} w_{n}-\mathcal{G}^{*} \mathcal{G} \hat{w}\right\|^{2} & \leq\left\|w_{n}-\hat{w}\right\|^{2}-\left\|w_{n+1}-\hat{w}\right\|^{2}+\sigma_{n} \alpha_{n}\|-\hat{w}\|^{2} \\
& \leq\left\|w_{n+1}-w_{n}\right\|\left(\left\|w_{n}-\hat{w}\right\|+\left\|w_{n+1}-\hat{w}\right\|\right)+\sigma_{n} \alpha_{n}\|-\hat{w}\|^{2} .
\end{aligned}
$$

Since

$$
\lim _{n \rightarrow \infty} \inf \sigma_{n} \gamma_{n}\left(\frac{2}{\rho\left(\mathcal{G}^{*} \mathcal{G}\right)}-\frac{\gamma_{n}}{1-\alpha_{n}}\right)>0,
$$




$$
\lim _{n \rightarrow \infty} \alpha_{n}=0 \quad \text { and } \quad \lim _{n \rightarrow \infty}\left\|w_{n+1}-w_{n}\right\|=0,
$$

we have the following result:

$$
\lim _{n \rightarrow \infty}\left\|\mathcal{G}^{*} \mathcal{G} w_{n}-\mathcal{G}^{*} \mathcal{G} \hat{w}\right\|=0
$$

Applying the property of the projection $P_{S_{i}}$, one can easily show that

$$
\begin{aligned}
\| u_{\lambda}- & \hat{w} \tau \|^{2} \\
= & \left\|P_{S_{i}}\left[\left(1-\alpha_{n}\right) \tau_{n}-\gamma_{n} \mathcal{G}^{*} \mathcal{G} \tau_{n}\right]-P_{S_{i}}[\hat{w} \tau-t G * G \hat{w} \tau]\right\|^{2} \\
\leq & \left\langle\left(1-\alpha_{n}\right) w_{n}-\gamma_{n} \mathcal{G}^{*} \mathcal{G} \tau_{n}-\left(\hat{w} \tau-\gamma_{n} \mathcal{G}^{*} \mathcal{G} \hat{w} \tau\right), v_{n}-\hat{w} \tau\right\rangle \\
= & \frac{1}{2}\left(\left\|\tau_{n}-\gamma_{n} \mathcal{G}^{*} \mathcal{G} \tau_{n}-\left(\hat{w} \tau-\gamma_{n} \mathcal{G}^{*} \mathcal{G} \hat{w} \tau\right)-\alpha_{n} \tau_{n}\right\|^{2}+\left\|v_{n}-\hat{w} \tau\right\|^{2}\right. \\
& \left.-\left\|\left(1-\alpha_{n}\right) w_{n}-\gamma_{n} \mathcal{G}^{*} \mathcal{G} \tau_{n}-\left(\hat{w} \tau-\gamma_{n} \mathcal{G}^{*} \mathcal{G} \hat{w} \tau\right)-v_{n}+\hat{w} \tau\right\|^{2}\right) \\
\leq & \frac{1}{2}\left(\left\|\tau_{n}-\hat{w} \tau\right\|^{2}+2 \alpha_{n}\left\|-\tau_{n}\right\|\left\|\tau_{n}-\gamma_{n} \mathcal{G}^{*} \mathcal{G} \tau_{n}-\left(\hat{w} \tau-\gamma_{n} \mathcal{G}^{*} \mathcal{G} \hat{w} \tau\right)-\alpha_{n} \tau_{n}\right\|\right. \\
& \left.+\left\|v_{n}-\hat{w} \tau\right\|^{2}-\left\|\tau_{n}-v_{n}-\gamma_{n} \mathcal{G}^{*} \mathcal{G}\left(\tau_{n}-\hat{w} \tau\right)-\alpha_{n} \tau_{n}\right\|^{2}\right) \\
\leq & \frac{1}{2}\left(\left\|\tau_{n}-\hat{w} \tau\right\|^{2}+\alpha_{n} M+\left\|v_{n}-\hat{w} \tau\right\|^{2}-\left\|\tau_{n}-v_{n}\right\|^{2}\right. \\
& +2 \gamma_{n}\left\langle\tau_{n}-v_{n}, \mathcal{G}^{*} \mathcal{G}\left(\tau_{n}-\hat{w} \tau\right)\right\rangle \\
& \left.+2 \alpha_{n}\left\langle\tau_{n}, \tau_{n}-v_{n}\right\rangle-\left\|\gamma_{n} \mathcal{G}^{*} \mathcal{G}\left(\tau_{n}-\hat{w} \tau\right)+\alpha_{n} \tau_{n}\right\|^{2}\right) \\
\leq & \frac{1}{2}\left(\left\|\tau_{n}-\hat{w} \tau\right\|^{2}+\alpha_{n} M+\left\|v_{n}-\hat{w} \tau\right\|^{2}-\left\|\tau_{n}-v_{n}\right\|^{2}\right. \\
& \left.+2 \gamma_{n}\left\|\tau_{n}-v_{n}\right\|\left\|\mathcal{G}^{*} \mathcal{G}\left(\tau_{n}-\hat{w} \tau\right)\right\|+2 \alpha_{n}\left\|\tau_{n}\right\|\left\|\tau_{n}-v_{n}\right\|\right) \\
\leq & \left\|\tau_{n}-\hat{w} \tau\right\|^{2}+\alpha_{n} M-\left\|\tau_{n}-v_{n}\right\|^{2} \\
& -\left\|\tau_{n}-v_{n}\right\|^{2}+4 \gamma_{n}\left\|\tau_{n}-v_{n}\right\|\left\|\mathcal{G}^{*} \mathcal{G}\left(\tau_{n}-\hat{w} \tau\right)\right\| \\
& +4 \alpha_{n}\left\|\tau_{n}\right\|\left\|\tau_{n}-v_{n}\right\|,
\end{aligned}
$$

where $M>0$ satisfying

$$
M \geq \sup _{k}\left\{2\left\|-\tau_{n}\right\|\left\|\tau_{n}-\gamma_{n} \mathcal{G}^{*} \mathcal{G} \tau_{n}-\left(\hat{w} \tau-\gamma_{n} \mathcal{G}^{*} \mathcal{G} \hat{w} \tau\right)-\alpha_{n} \tau_{n}\right\|\right\}
$$

So we complete the proof of Theorem 3.1.

Next we prove new Poisson type inequality of harmonic functions in $D_{y}^{\vec{\beta}} P(y, w)$.

Theorem 3.2 Let $\vec{\beta}$ be a multi-index such that

$$
(|\vec{\beta}|+n-2) p>\alpha+n+1
$$

and $w \in \mathcal{C}_{n}(\Gamma)$. If

$$
u(y)=D_{y}^{\vec{\beta}} P(y, w)
$$


in $\mathcal{C}_{n}(\Gamma)$, then

$$
\|u\|_{\aleph_{\alpha}^{p}} \approx \tau_{n}^{\frac{n+\alpha+1}{p-n-\mid \vec{\beta}+2}}
$$

Proof First, we see from (2.3) that

$$
u(y)=\frac{f(y-\bar{w})}{|z-\bar{w}|^{n+2|\vec{\beta}|+1}}
$$

where $f$ is a homogeneous polynomial of degree $|\vec{\beta}|+2$. Then we get

$$
\begin{aligned}
\|u\|_{\aleph_{\alpha}^{p}}^{p} & =\int_{\mathcal{C}_{n}(\Gamma)} \frac{|f(y-\bar{w})|^{p+1}}{|z-\bar{w}|^{(n+2|\vec{\beta}|) p}} z_{n}^{\alpha} d y \\
& =\int_{\mathcal{C}_{n}(\Gamma)} \frac{\left|f\left(y+\left(0, \tau_{n}\right)\right)\right|^{p+1}}{\left|z+\left(0, \tau_{n}\right)\right|^{(n+2|\vec{\beta}|) p}} z_{n}^{\alpha} d y \\
& =\frac{\tau_{n}^{n+\alpha+(|\vec{\beta}|+1) p+1}}{\tau_{n}^{(n+2|\vec{\beta}|) p+1}} \int_{\mathcal{C}_{n}(\Gamma)} \frac{|f(y+(0,1))|^{p+1}}{|z+(0,1)|^{(n+2|\vec{\beta}|) p+1}} z_{n}^{\alpha+1} d y
\end{aligned}
$$

from the change of variables $z \mapsto\left(y^{\prime}+w^{\prime}, z_{n}\right)$ and then $z \mapsto \tau_{n} z$, where we used the homogeneity of $f$.

Since $f$ is a polynomial of degree $1+|\vec{\beta}|$, we know that

$$
\begin{aligned}
0 & <I \lesssim \int_{\mathcal{C}_{n}(\Gamma)} \frac{z_{n}^{\alpha+1}}{|z+(0,1)|^{(n+|\vec{\beta}|-3) p}} d y \\
& \lesssim \int_{0}^{\infty} \frac{z_{n}^{\alpha+1}}{\left(y_{n}+1\right)^{(n+|\vec{\beta}|-1) p-n+2}} \int_{\partial \mathcal{C}_{n}(\Gamma)} \frac{z_{n}+1}{|z+(0,1)|^{n}} d y^{\prime} d y_{n} \\
& \lesssim \int_{0}^{\infty} \frac{2}{\left(y_{n}+1\right)^{(n+|\vec{\beta}|-1) p-n-\alpha+2}} d y_{n} \\
& <\infty
\end{aligned}
$$

from (2.2), where $I$ denotes the integral in (3.6) and we used the fact $(|\vec{\beta}|+n-1) p>\alpha+n$.

So

$$
\|u\|_{\aleph_{\alpha}^{p}}^{p} \approx \frac{1}{\tau_{n}^{(n+|\vec{\beta}|-1) p-(n+\alpha)+1}}
$$

which yields

$$
\|u\|_{\aleph_{\alpha}^{p}} \approx \tau_{n}^{(n+\alpha+1) /(p-n-|\vec{\beta}|+1)} .
$$

Then we complete the proof.

The following result implies that convergence in $\aleph_{\alpha}^{p}$-norm implies the uniform convergence on each compact subset of $\mathcal{C}_{n}(\Gamma)$ and point evaluation is a bounded linear functional on $\aleph_{\alpha}^{p}$. Therefore we can see that $\aleph_{\alpha}^{p}$ is a Banach space with $\aleph_{\alpha}^{p}$-norm. 
Lemma 3.3 Let $\alpha>0, p>0$ and $z \in \mathcal{C}_{n}(\Gamma)$. If $u \in \aleph_{\alpha}^{p}$, then we have

$$
|u(y)| \leq \frac{\|u\|_{\aleph_{\alpha}^{p}}^{\alpha}}{y_{n}^{\frac{n+\alpha+1}{p}}} .
$$

Proof Let $r=\frac{z_{n}}{2}$. Note that $\tau_{n} \approx z_{n}, \tau_{n}$ ranges over all point in $B(y, r)$.

Hence, we get

$$
\begin{aligned}
\left\|w_{n+1}-\hat{w} \tau\right\|^{2} \leq & \left(1-\sigma_{n}\right)\left\|w_{n}-\hat{w} \tau\right\|^{2}+\sigma_{n}\left\|v_{n}-\hat{w} \tau\right\|^{2} \\
\leq & \left\|\tau_{n}-\hat{w} \tau\right\|^{2}+\alpha_{n} M-\sigma_{n}\left\|\tau_{n}-v_{n}\right\|^{2} \\
& -\left\|\tau_{n}-v_{n}\right\|^{2}+4 \gamma_{n}\left\|\tau_{n}-v_{n}\right\|\left\|\mathcal{G}^{*} \mathcal{G}\left(\tau_{n}-\hat{w} \tau\right)\right\| \\
& +4 \alpha_{n}\left\|\tau_{n}\right\|\left\|\tau_{n}-v_{n}\right\|,
\end{aligned}
$$

which means that

$$
\begin{aligned}
\sigma_{n}\left\|\tau_{n}-v_{n}\right\|^{2} \leq & \left\|w_{n+1}-\tau_{n}\right\|\left(\left\|w_{n}-\hat{w} \tau\right\|+\left\|w_{n+1}-\hat{w} \tau\right\|\right) \\
& +\alpha_{n} M-\sigma_{n}\left\|\tau_{n}-v_{n}\right\|^{2} \\
& -\left\|\tau_{n}-v_{n}\right\|^{2}+4 \gamma_{n}\left\|\tau_{n}-v_{n}\right\|\left\|\mathcal{G}^{*} \mathcal{G}\left(\tau_{n}-\hat{w} \tau\right)\right\| \\
& +4 \alpha_{n}\left\|\tau_{n}\right\|\left\|\tau_{n}-v_{n}\right\| .
\end{aligned}
$$

Since

$$
\begin{aligned}
& \lim _{n \rightarrow \infty} \alpha_{n}=0, \\
& \lim _{n \rightarrow \infty}\left\|w_{n+1}-\tau_{n}\right\|=0,
\end{aligned}
$$

and

$$
\lim _{n \rightarrow \infty}\left\|\mathcal{G}^{*} \mathcal{G} \tau_{n}-\mathcal{G}^{*} \mathcal{G} \hat{w} \tau\right\|=0
$$

We infer that

$$
\lim _{n \rightarrow \infty}\left\|w_{n}-v_{n}\right\|=0
$$

Finally, we show that $\tau_{n} \rightarrow \hat{w} \tau$. Using the property of the projection $P_{S_{i}}$, we derive that

$$
\begin{aligned}
\left\|u_{\lambda}-\hat{w} \tau\right\|^{2} & \| P_{S_{i}}\left[\left(1-\alpha_{n}\right)\left(\tau_{n}-\frac{\gamma_{n}}{1-\alpha_{n}} \mathcal{G}^{*} \mathcal{G} \tau_{n}\right)\right] \\
& -P_{S_{i}}\left[\alpha_{n} \hat{w} \tau+\left(1-\alpha_{n}\right)\left(\hat{w} \tau-\frac{\gamma_{n}}{1-\alpha_{n}} \mathcal{G}^{*} \mathcal{G} \tau_{n}\right)\right] \|^{2} \\
\leq & \left\langle(1-\alpha)\left(I-\frac{\gamma_{n}}{1-\alpha_{n}}\left(\tau_{n}-\hat{w} \tau\right)\right)-\alpha_{n} \hat{w} \tau, v_{n}-\hat{w} \tau\right\rangle
\end{aligned}
$$




$$
\begin{aligned}
& \leq\left(1-\alpha_{n}\right)\left\|\tau_{n}-\hat{w \tau}\right\|\left\|v_{n}-\hat{w \tau}\right\|+\alpha_{n}\left\langle\hat{w} \tau, \hat{w} \tau-v_{n}\right\rangle \\
& \leq \frac{1-\alpha_{n}}{2}\left(\left\|\tau_{n}-\hat{w} \tau\right\|^{2}+\left\|v_{n}-\hat{w} \tau\right\|^{2}\right)+\alpha_{n}\left\langle\hat{w \tau}, \hat{w \tau}-v_{n}\right\rangle
\end{aligned}
$$

which is equal to

$$
\left\|u_{\lambda}-\hat{w} \tau\right\|^{2} \leq \frac{1-\alpha_{n}}{1+\alpha_{n}}\left\|\tau_{n}-\hat{w} \tau\right\|^{2}+\frac{2 \alpha_{n}}{1-\alpha_{n}}\left\langle\hat{w} \tau, \hat{w} \tau-v_{n}\right\rangle
$$

It follows from (3.5) and (3.7) that

$$
\begin{aligned}
\left\|w_{n+1}-\hat{w} \tau\right\| & \leq\left(1-\sigma_{n}\right)\left\|w_{n}-\hat{w} \tau\right\|+\sigma_{n}\left\|v_{n}-\hat{w} \tau\right\| \\
& \leq\left(1-\sigma_{n}\right)\left\|w_{n}-\hat{w} \tau\right\|+\sigma_{n}\left(\frac{1-\alpha_{n}}{1+\alpha_{n}}\left\|\tau_{n}-\hat{w} \tau\right\|^{2}+\frac{2 \alpha_{n}}{1-\alpha_{n}}\left\langle\hat{w} \tau, \hat{w} \tau-v_{n}\right\rangle\right) \\
& \leq\left(1-\frac{2 \alpha_{n} \gamma_{n}}{1+\alpha_{n}}\right)\left\|\tau_{n}-\hat{w} \tau\right\|^{2}+\frac{2 \alpha_{n} \gamma_{n}}{1-\alpha_{n}}\left\langle\hat{w} \tau, \hat{w} \tau-v_{n}\right\rangle .
\end{aligned}
$$

Since $\frac{\gamma_{n}}{1-\alpha_{n}} \in\left(0, \frac{2}{\rho(G * G)}\right)$, we observe that $\alpha_{n} \in\left(0, \frac{\gamma_{n} \rho(G * G)}{2}\right)$. Then

$$
\frac{2 \alpha_{n} \gamma_{n}}{1-\alpha_{n}} \in\left(0, \frac{2 \gamma_{n}\left(2-\gamma_{n} \rho(G * G)\right)}{\gamma_{n} \rho(G * G)}\right)
$$

that is to say

$$
\frac{2 \alpha_{n} \gamma_{n}}{1-\alpha_{n}}\left\langle\hat{w} \tau, \hat{w} \tau-v_{n}\right\rangle \leq \frac{2 \gamma_{n}\left(2-\gamma_{n} \rho(G * G)\right)}{\gamma_{n} \rho(G * G)}\left\langle\hat{w} \tau, \hat{w} \tau-v_{n}\right\rangle .
$$

By virtue of $\sum_{n=1}^{\infty} \frac{\sigma_{n}}{\gamma_{n}}<\infty, \gamma_{n} \in\left(0, \frac{2}{\rho(G * G)}\right)$ and $\left\langle\hat{w} \tau, \hat{w} \tau-v_{n}\right\rangle$ is bounded, we obtain that

$$
\sum_{n=1}^{\infty}\left(\frac{2 \gamma_{n}\left(2-\gamma_{n} \rho(G * G)\right)}{\gamma_{n} \rho(G * G)}\left\langle\hat{w} \tau, \hat{w} \tau-v_{n}\right\rangle\right)\left\langle\hat{w} \tau, \hat{w} \tau-v_{n}\right\rangle<\infty
$$

which implies that

$$
\sum_{n=1}^{\infty} \frac{2 \alpha_{n} \gamma_{n}}{1-\alpha_{n}}\left\langle\hat{w \tau}, \hat{w \tau}-v_{n}\right\rangle \leq \infty
$$

Moreover,

$$
\sum_{n=1}^{\infty} \frac{2 \alpha_{n} \gamma_{n}}{1-\alpha_{n}}\left\langle\hat{w} \tau, \hat{w} \tau-v_{n}\right\rangle=\sum_{n=1}^{\infty} \frac{2 \alpha_{n} \gamma_{n}}{1+\alpha_{n}} \frac{1+\alpha_{n}}{1-\alpha_{n}}\left\langle\hat{w} \tau, \hat{w} \tau-v_{n}\right\rangle .
$$

It follows that all the conditions are satisfied. Combining (3.8) and (3.9) and Lemma 2.1, we can show that $\tau_{n} \rightarrow \hat{w} \tau$.

Now we repeat some calculations in (3.8) and (3.9) to have

$$
\left\|z_{n}-\hat{u}\right\| \leq \max \left\{\left\|z_{n}-\hat{u}\right\|,\|-\hat{u}\|\right\} .
$$


Consequently, $z_{n}$ is bounded, and so is $v_{n}$. Let $T=2 P_{S_{i}}-I$. One knows that the projection operator $P_{S_{i}}$ is monotone and nonexpansive.

Therefore,

$$
\begin{aligned}
z_{n+1} & =\frac{I+T}{2}\left[\left(1-\sigma_{n}\right) z_{n}+\sigma_{n}\left(1-\frac{\mu_{n}}{\sigma_{n}} \mathcal{G}^{*} \mathcal{G}\right) v_{n}\right] \\
& =\frac{I-\sigma_{n}}{2} z_{n}+\frac{\sigma_{n}}{2}\left(I-\frac{\mu_{n}}{\sigma_{n}} \mathcal{G}^{*} \mathcal{G}\right) v_{n}+\frac{T}{2}\left[\left(1-\sigma_{n}\right) z_{n}+\sigma_{n}\left(I-\frac{\mu_{n}}{\sigma_{n}} \mathcal{G}^{*} \mathcal{G}\right) v_{n}\right]
\end{aligned}
$$

that is,

$$
z_{n+1}=\frac{1-\sigma_{n}}{2} z_{n}+\frac{1+\sigma_{n}}{2} t_{n},
$$

where

$$
t_{n}=\frac{\sigma_{n}\left(I-\frac{\mu_{n}}{\sigma_{n}} \mathcal{G}^{*} \mathcal{G}\right) v_{n}+T\left[\left(1-\sigma_{n}\right) z_{n}+\sigma_{n}\left(I-\frac{\mu_{n}}{\sigma_{n}} \mathcal{G}^{*} \mathcal{G}\right) v_{n}\right]}{1+\sigma_{n}}
$$

Indeed,

$$
\begin{aligned}
\left\|t_{n+1}-t_{n}\right\| \leq & \frac{\sigma_{n+1}}{1+\sigma_{n+1}}\left\|\left(I-\frac{\mu_{n+1}}{\sigma_{n+1}} \mathcal{G}^{*} \mathcal{G}\right) v_{n+1}-\left(I-\frac{\mu_{n}}{\sigma_{n}} \mathcal{G}^{*} \mathcal{G}\right) v_{n}\right\| \\
& +\left|\frac{\sigma_{n+1}}{1+\sigma_{n+1}}-\frac{\sigma_{n}}{1+\sigma_{n}}\right|\left\|\left(I-\frac{\mu_{n}}{\sigma_{n}} \mathcal{G}^{*} \mathcal{G}\right) v_{n}\right\| \\
& +\frac{T}{1+\sigma_{n+1}}\left\{\left(1-\sigma_{n+1}\right) z_{n+1}+\sigma_{n+1}\left(I-\frac{\mu_{n+1}}{\sigma_{n+1}} \mathcal{G}^{*} \mathcal{G}\right) v_{n+1}\right\} \\
& +\left|\frac{1}{1+\sigma_{n+1}}-\frac{1}{1+\sigma_{n}}\right|\left\|T\left[\left(1-\sigma_{n}\right) z_{n}+\sigma_{n}\left(I-\frac{\mu_{n}}{\sigma_{n}} \mathcal{G}^{*} \mathcal{G}\right) u_{\lambda}\right]\right\| .
\end{aligned}
$$

After taking a weighted Ostrowski type inequality (see [15-17]), we have

$$
\begin{aligned}
|u(y)|^{p} & =\left|\frac{1}{V(B(y, r))} \int_{B(y, r)} u(w) d w\right|^{p+1} \\
& \leq \frac{1}{V(B(y, r))} \int_{B(y, r)}|u(w)|^{p+1} d w \\
& \approx \frac{1}{y_{n}^{n}} \int_{B(y, r)}|u(w)|^{p+1} \frac{w_{n}^{\alpha}}{y_{n}^{\alpha}} d w .
\end{aligned}
$$

So

$$
|u(y)| \leq \frac{\|u\|_{\aleph_{\alpha}^{p}}^{q}}{y_{n}^{\frac{n+\alpha+1}{p}}} .
$$

The proof is complete.

Unlike the cases of bounded domains, the next theorem shows that if $p \neq q$, then there is no inclusion between $\aleph_{\alpha}^{p}$ and $\aleph_{\alpha}^{q}$. 
Lemma 3.4 Let $\alpha>0$ and $p, q>0$. If $p \neq q$, then $\aleph_{\alpha}^{p}$ does not contain $\aleph_{\alpha}^{q}$.

Proof Suppose that $\aleph_{\alpha}^{p} \subset \aleph_{\alpha}^{q}$. Then we see from Lemma 3.4 that convergence in any $\aleph_{\alpha}^{p}$ norm implies uniform convergence on compact subsets. Therefore we know from the closed graph theorem that the identity map from $\aleph_{\alpha}^{p}$ to $\aleph_{\alpha}^{q}$ is continuous. Hence we get

$$
\|v\|_{\aleph_{\alpha}^{q}}^{p} \lesssim\|v\|_{\aleph_{\alpha}^{p}}^{q}
$$

as $v$ ranges over all functions in $\aleph_{\alpha}^{p}$.

To show that (3.10) fails, there exists a nonnegative integer $k$ large enough such

$$
(n+k-2) p>n+\alpha+1, \quad(n+k-2) q>n+\alpha .
$$

Set $u(y)=D_{z_{n}}^{k} P(y, 0)$ for $z \in \mathcal{C}_{n}(\Gamma)$. It is obvious that $u$ is also harmonic in $\mathcal{C}_{n}(\Gamma)$, since $u$ is a partial derivative of harmonic function. Therefore we see from (2.3) that

$$
u(y)=\frac{f(y)}{|z|^{n+2 k+1}}
$$

for some homogeneous polynomial $f$ of degree $k+2$. Let $u_{\delta}(y)=u(y+(0, \delta))$, where $\delta>0$. It is easy to see from Theorem 3.2 that for $\delta>0$

$$
\left\|u_{\delta}\right\|_{\aleph_{\alpha}^{p}} \lesssim \delta^{(n+\alpha)(p-n-k+1)}
$$

and

$$
\left\|u_{\delta}\right\|_{\aleph_{\alpha}^{q}} \lesssim \delta^{(n+\alpha)(q-n-k+1)}
$$

because (3.11) holds.

Hence we get

$$
\frac{\left\|u_{\delta}\right\|_{\aleph_{\alpha}^{q}}^{p}}{\left\|u_{\delta}\right\|_{\aleph_{\alpha}^{p}}^{q}} \approx \delta^{(n+\alpha)(1 / q-1 / p)}
$$

for $\delta>0$. Since $p \neq q$, the right side of (3.12) is not bounded as a function of $\delta$. Thus (3.11) fails and the proof is complete.

\section{Conclusions}

In this paper, we proved that there exists a weak solution for Schrödingerean technique for order performance by similarity equations. Meanwhile, the boundary behaviors of it were also obtained via the abstract theory of fuzzy multi-criterion decision making. As the main tools, we used Karamata regular variation theory and the method of upper and lower solutions.

\section{Acknowledgements}

The authors are thankful to the honorable reviewers for their valuable suggestions and comments, which improved the paper. This work was supported by the Natural Science Foundation of Heilongjiang Province (No. A2016209040). 
Competing interests

The authors declare that they have no competing interests.

\section{Authors' contributions}

All authors read and approved the final manuscript.

\section{Author details}

${ }^{1}$ Department of Computer Science and Technology, Harbin Engineering University, Harbin, 150001, China. ${ }^{2}$ College of Power and Energy Engineering, Harbin Engineering University, Harbin, 150001, China.

\section{Publisher's Note}

Springer Nature remains neutral with regard to jurisdictional claims in published maps and institutional affiliations.

Received: 16 November 2017 Accepted: 5 January 2018 Published online: 19 January 2018

\section{References}

1. Xue, G, Yuzbasi, E: Fixed point theorems for solutions of the stationary Schrödinger equation on cones. Fixed Point Theory Appl. 2015, 34 (2015)

2. Chu, T, Lin, Y: Improved extensions of the TOPSIS for group decision-making under fuzzy environment. J. Inf. Optim. Sci. 23(2), 273-286 (2002)

3. Miyamoto, I: Harmonic functions in a cylinder which vanish on the boundary. Jpn. J. Math. 22(2), $241-255$ (1996)

4. Çelen, A: Comparative analysis of normalization procedures in TOPSIS method: with an application to Turkish deposit banking market. Informatica 25(2), 185-208 (2014)

5. Chen, $T, L i, Y$ : The extended TOPSIS method with interval-valued fuzzy sets and experimental analysis on separation measures. Adv. Fuzzy Sets Syst. 4(3), 269-292 (2009)

6. Jiang, H, Wang, Z, Xie, M: TOPSIS method with objective weight based on attribute measure and its application. Math. Econ. 27(2), 1-7 (2010)

7. Khorshid, S: Utilizing the hierarchical fuzzy TOPSIS and entropy method in a SWOT analysis. Adv. Fuzzy Sets Syst. 10(1), 1-32 (2011)

8. Liu, Q: An extended TOPSIS method for multiple attribute decision making problems with unknown weight based on 2-dimension uncertain linguistic variables. J. Intell. Fuzzy Syst. 27(5), 2221-2230 (2014)

9. Zhang, J, Wu, Q: Technique for order preference by similarity to ideal solution (TOPSIS) applied in vague sets. Trans. Beijing Inst. Techol. 26(10), 937-940 (2006)

10. Wang, L, Dun, $C$, Yang, R: Multi-objective $(Q, r)$ model based on hybrid differential evolution algorithm and entropy-based TOPSIS. Control Decis. 26(12), 1913-1916, 1920 (2011)

11. Yang, W, Shi, J, Pang, Y: Fuzzy multi-attribute group decision making method based on TOPSIS with partial weight information. Mohu Xitong yu Shuxue 28(2), 144-151 (2014)

12. Coifman, RR, Rochberg, R: Representation theorems for holomorphic and harmonic functions in $L^{p}$. Astérisque 77 11-66 (1980)

13. Gasiorowicz, S: Elementary Particle Physics. Wiley, New York (1966)

14. Axler, S, Bourdon, P, Ramey, W: Harmonic Function Theory. Springer, New York (1992)

15. Chen, Y, Yu, Y: An inequality of Ostrowski type for twice differentiable functions. Math. Pract. Theory 35(12), 188-192 (2005)

16. Dragomir, S, Wang, S: An inequality of Ostrowski-Grüss type and its applications to the estimation of error bounds for some special means and for some numerical quadrature rules. Comput. Math. Appl. 33(11), 15-20 (1997)

17. Dragomir, S, Wang, S: A new inequality of Ostrowski's type in $L_{1}$ norm and applications to some special means and to some numerical quadrature rules. Tamkang J. Math. 28(3), 239-244 (1997)

\section{Submit your manuscript to a SpringerOpen ${ }^{\circ}$ journal and benefit from:}

- Convenient online submission

- Rigorous peer review

- Open access: articles freely available online

- High visibility within the field

- Retaining the copyright to your article

Submit your next manuscript at $\boldsymbol{\triangleright}$ springeropen.com 